\title{
Factor-GARCH Modeling of the Treasury Term Structures
}

\author{
Christopher F. Baum \\ Department of Economics \\ Boston College \\ Chestnut Hill MA 02167
}

\author{
Basma Bekdache \\ Department of Economics \\ Wayne State University \\ Detroit MI 48202
}

June 1996

\section{Introduction}

In this paper, we test the multivariate model of securities' excess returns formulated by Engle et al. (1990) on an expanded set of maturities. By applying their methodology to the entire Treasury term structure, we consider the applicability of a parsimonious common factor approach to the dynamics of short-, medium-, and long-term interest rates. We extend their methodology to incorporate asymmetric GARCH representations, in which the slope of the yield curve (and its sign) affects the evolution of the conditional variance of excess returns in fixed-income and equity markets. We find this approach quite successful in explaining the comovements of excess returns on the spectrum of Treasury issues for the 1962-1992 period.

The paper is organized as follows. In the first section, we present some stylized facts about the postwar term structure, making use of Coleman et al.'s (1993) estimates of spot yields to construct holding period returns and excess returns vis-àvis the return on a one-month security. We then present similar evidence on the series' second moments. In contrast to many studies which have focused solely on money-market yields, we consider the entire Treasury term structure, so that we may make inferences about the behavior of capital market rates, and the interactions between short- and long-term fixed income returns and equity returns. Section 2 presents an explicit model of the second moments of excess returns, applying the Factor GARCH specification of Engle et al.'s no-arbitrage term structure model (1990) to the entire Treasury term structure and equity returns. In the last section, we make some summary remarks.

$\S$ We acknowledge the comments of John Barkoulas and participants at the 1995 Conference of the Society for Computational Economics. The usual disclaimer applies. 


\section{Stylized Facts about the Postwar Term Structure of Treasury Rates}

A very large proportion of empirical term structure models have been applied to the observable spot yields in the U.S. Treasury bill market-that is, yields for zero to 12 months' tenor discount bills, which are readily available from CRSP as the "Fama files." In this study, we consider the entire Treasury term structure-for bills, notes, and bonds-so that both money market and capital market returns may be modeled. We make use of a set of monthly estimates of Treasury market spot yields constructed from coupon securities' quotations by Coleman et al. (1993, CFI).

Our work is based on the spot yields for the 14 specific tenors analyzed by CFI for their sample period of 1955 through 1992,1 transforming them into estimated one-month holding period returns. ${ }^{2}$ We model excess return series, created by subtracting the annualized holding period return on a one-month Treasury from the holding period return for each longer tenor. Taking the one-month security (the shortest maturity reported by $\mathrm{CFI}$ ) as the risk-free rate, the resulting excess return series show the compensation that has been earned, ex post, by those who have assumed the risks associated with holding longer maturity bonds. These excess returns are not necessarily increasing in tenor. We also consider the variance in these excess returns series, and how it is changing over time. A moving-window estimate of variance (with a window of 12 months' width) was calculated for each excess returns series. Analysis of these estimates reveals that the variability of excess returns increased dramatically in the 1980s when the Federal Reserve deemphasized interest rate targeting.

The correlations between excess returns on short-term securities and excess returns on longer-term securities vary considerably over the sample. One-, two- and five-year securities' excess returns were quite highly correlated to excess returns on the three-month security until the mid-1980s. Returns on the long-term (20 year tenor) security were much more weakly related to those on three-month bills, but vary from below 0.20 to nearly 0.60 over the period. In Figure 1, we present estimates of correlations between the three-month excess returns series and those of 12

\footnotetext{
${ }^{1}$ We do not consider their "long" tenor, as it does not correspond to a fixed maturity.

2 The discrete tenors in CFI's study do not allow us to derive holding period returns (as one could from the Fama Treasury bill files). We thus constructed hypothetical spot yields for the missing tenors as linear interpolations of the CFI spot yields.
} 
months, 2 years, 5 years, and 20 years. ${ }^{3}$ These movements suggest that excess returns are quite strongly interrelated at all tenors, reflecting the comovements of risk premia on the various tenors' yields, and lends further support to the concept of a common-factor-based modelling strategy.

These stylized facts about the postwar Treasury term structure suggest that a workable term structure model should explicitly consider time variation in the second moments of residual series as well as capture the interaction among tenors. The following section presents such a model in which we have implemented time variation in the second moments, as well as asymmetry in the modelled conditional variances, using the approach of Gourieroux and Monfort (1992).

\section{Estimates of Factor-GARCH models for the Treasury term structure}

Term structure modelling has followed two broad strands of development: general equilibrium models, such as those pioneered by Cox, Ingersoll and Ross (1985), and no-arbitrage partial equilibrium models. In this paper, we consider a model of the latter genre, developed by Engle et al. (1990), and extend it to the consideration of the complete Treasury term structure rather than just its short end. In this framework, we consider whether time-varying volatility in asset returns is a meaningful determinant of excess returns in the medium and long-term sectors of the Treasury market. The term structure literature contains scattered evidence that conclusions drawn from Treasury bill data do not readily extend to the medium and long term sectors of the Treasury market. For instance, Engsted and Tangaard (1994) extend the work of Hall, Anderson and Granger (1992) and study the cointegration properties of the term structure of interest rates, using 2-, 5-, and 10-year yields from McCulloch and Kwon's (1993) data. They find that the breakdown in the cointegrating relationship between short rates that occurs during the 1979-1982 Federal Reserve operating policy shift does not appear in the longer maturity term structure. This suggests that risk premia behave differently over the maturity structure, and that the explanation that term premia become nonstationary with a regime shift may not hold true for longer maturities.

Similar evidence is found in a study by Froot (1989), where survey data on interest rate expectations are used to study the relative importance of time-varying term premia and expectational errors in explaining rejections of the pure

3 The moving correlations are computed annually from 36 monthly observations; the date on the horizontal axis is the left endpoint of the three-year span. 
expectations theory. He finds that average expected excess holding period returns increase with maturity, and that term premia on long term bonds are more volatile than those of shorter tenors. Canova and Marrinan (1995) study excess returns on five-year securities generated by one- and three-month holding periods, and find that one- and three-month excess returns series have different statistical properties. They conclude that there are "nontrivial differences in the risk characteristics in agents investing at different maturities" (p. 64) and suggest that a segmented markets approach may be warranted.

Engle et al. (1990, henceforth ENR) argue that a multivariate approach to the modelling of asset returns is clearly justified, since even in static asset pricing models, the full covariance matrix of asset returns is required to derive estimates of a single asset's risk premium. The implication for dynamic modeling of the term structure becomes an empirical challenge, as allowing for temporal heteroskedasticity in this covariance matrix will imply, for instance, a very high dimensional multivariate ARCH model. If the number of assets is kept small, the ability to incorporate these cross-market effects is hindered; yet the number of timevarying covariances to be modelled grows rapidly with the number of assets. ENR deal with this dilemma by proposing the use of Engle's (1987) Factor-ARCH specification, which provides a parsimonious structure for the conditional covariance matrix of asset returns, rendering the problem tractable for a sizable number of assets. In this section, we sketch their approach, which we then apply to the full term structure of Treasury returns.

ENR model the $\mathrm{N}$-vector $\mathrm{y}_{\mathrm{t}}$ of asset excess returns as dependent on a mean vector $\mu_{t}$ and a time-varying covariance matrix $H_{t}$, where $H$ is then expressed (ENR (1)) as

$$
H_{t}=\sum_{k=1}^{K} \beta_{k} \beta^{\prime}{ }_{k} \lambda_{k t}+\Omega
$$

where $\mathrm{K}$, the number of common factors driving the covariance matrix, is presumably much smaller than $\mathrm{N}$, and $\Omega$ is an $\mathrm{NxN}$ positive semi-definite matrix. They illustrate that this model may be related to Ross' (1976) arbitrage pricing theory factor model. To implement the model, it is necessary to replace the unobservable factors $\lambda_{\mathrm{kt}}$ with measures of the conditional variances of some "factor-representing" portfolios of the assets, $\theta_{\mathrm{kt}}$, which are by construction perfectly correlated with the latent variables $\lambda$. In ENR's application to twelve maturities of Treasury bills and a stockmarket index, they choose two factors-an arbitrarily weighted bond portfolio and a pure stock portfolio-as sufficient, and apply a recursive representation where 
the stockmarket portfolio's excess returns are generated in a univariate model, but the bond portfolio's excess returns depend as well on the stockmarket portfolio's behavior.

\subsection{Excess returns for Treasury and stockmarket index portfolios}

We use the Coleman et al. (1993, henceforth CFI) yields to construct estimates of one-month holding period returns. Comparable returns on a diversified stock portfolio are derived from the CRSP value-weighted index for the NYSE/AMEX, which is first available in July 1962. Our sample for this section of the paper thus extends from that date through December 1992, the terminal point of CFI's study.

The one-month tenor in CFI's data is taken as an estimate of the riskless return, and excess return series for all tenors and the stockmarket index are constructed as the difference between their one-month holding period returns and the corresponding "riskless" return. Summary statistics for these one-month excess return series are given in Table 1. Mean excess returns are similar for the short- and medium-term tenors, but fall off sharply for 15 years, becoming negative at 20 years. Excess returns for the stockmarket index are almost four times larger, on average, than for any Treasury security. The variance of excess returns series grows markedly with maturity, surpassing that of the stockmarket index for the 15- and 20-year tenors. Treasury excess returns are positively skewed: sizably for the short-term tenors, but negatively for the stockmarket. All excess return series exhibit excess kurtosis, generally declining with tenor; the stockmarket series' kurtosis is smaller than that of any tenor. Significant serial correlation is found in almost every Treasury series, but is not present in either the stockmarket series or its square.

The last row of the table presents similar statistics for an equally-weighted portfolio of Treasury securities. Given the greater number of shorter maturities in the CFI data, this portfolio has a duration of 65 months, or almost 5.5 years. Mean excess returns for the Treasury portfolio are 8 basis points per month higher than the riskfree rate, which averages 46 basis points per month (5.66 per cent per annum). The equally-weighted bond portfolio would yield, on average, 6.69 per cent per annum, with a variance slightly less than that of a 48-month Treasury. The stock portfolio, by comparison, would yield 11.35 per cent per annum (inclusive of dividends), with a variance more than five times higher than that of the equallyweighted bond portfolio. 
A natural question in considering a set of highly correlated assets such as Treasury securities of different tenors is the extent to which a small number of common factors are driving their prices and yields. To gauge this commonality, we followed ENR's approach and performed principal components analysis on the sample covariance matrix of the Treasury and stockmarket excess returns series. The largest eigenvalue explains 77 per cent of the total variance, with the second explaining 14 per cent and the third an additional five per cent. Almost 97 per cent of the total variance is explained by the first three principal components. Although the number of significant eigenvalues does not specifically indicate the number of dynamic factors appropriate for our model, it would appear that a two-factor model might be able to capture the behavior of the excess returns series.

\subsection{Asymmetric GARCH models of portfolio excess returns}

Following Engle et al. (1990, p.223), we do not attempt to determine portfolio weights within the model, but rather specify weights for two factor-representing portfolios: an equally-weighted bond portfolio and a portfolio containing only the stockmarket index. As noted above, the set of tenors for Treasuries in our data imply that the equally-weighted bond portfolio will have risk characteristics approximately equal to a 5.5-year tenor security. In fitting a GARCH model to the portfolio excess returns series, we considered various asymmetric forms of the basic GARCH model. Other researchers (cf. Gourieroux and Monfort (1992)) have found support for asymmetries in either the mean equation or the conditional variance equation of the GARCH formulation. In the context of the factor-representing portfolios' excess returns, we might expect the conditional variance to respond differently to increases and decreases in risk. The estimates presented below are the outcome of a specification search over various forms of asymmetry.

In formulating a model for excess returns of the bond and stockmarket portfolios, we did not find support in the data for the GARCH-in-mean formulation used by ENR (p.223), in which the conditional mean of each portfolio's excess return series depends only on its conditional variance. Specifications of the conditional mean that included (current or lagged) excess returns on the alternate portfolio were more successful. Our results also exhibit the "causality in variance" (ENR, p. 224), but in a different direction: making use of an asymmetric response coefficient, we find that the sign of lagged excess returns in the bond equation plays a significant role in each portfolio's conditional variance equation. 
For the stockmarket portfolio, we found greatest support for a specification that includes lagged excess returns on the 20-year maturity "long bond." There appears to be meaningful asymmetry in the variance equation's intercept, depending on the sign of lagged excess returns on the bond portfolio. This asymmetry was captured by a dummy, $D_{B 240}$, which takes on a value of unity when lagged excess returns on the bond portfolio are positive. Implementation of the GARCH $(1,1)$ model for the stockmarket index portfolio via RATS' BHHH algorithm yields (standard errors in parentheses):

$$
\begin{aligned}
& R_{\text {Stock }_{t}}=\underset{(0.156)}{0.447}+\underset{(0.021)}{0.116} R_{B 240_{t-1}}+u_{\text {Stock }_{t}} \\
& \theta_{\text {Stock }_{t}}=\underset{(0.034)}{1.185}+\underset{(0.050)}{1.918} D_{B 240_{t-1}}+\underset{(0.002)}{0.129} u_{\text {Stock }_{t-1}^{2}}+\underset{(0.009)}{0.798} \theta_{\text {Stock }_{t-1}}
\end{aligned}
$$

where $R_{\text {Stock }}$ is the excess returns series for the stockmarket portfolio. The asymmetry in the variance equation suggests that the conditional variance, $\theta_{\text {Stock }_{t}}$, increases markedly when bond excess returns have been positive: that is, when the yield curve is positively sloped. This is appealing, in that it suggests that a higher (lower) level of risk in the bond market-as reflected in higher (lower) term premiawill increase (decrease) volatility in the stockmarket, as the markets (and their respective derivative markets) act to equalize the "market price of risk" across fixedincome and equity venues.

The estimated model for the stockmarket index exhibits a strong interaction between lagged bond returns and current returns in the stockmarket. The persistence of the $\mathrm{ARCH}$ effect is quite strong in the variance equation. Ljung-Box $\mathrm{Q}$ statistics for 12 autocorrelations of the residuals and their squares are 9.22 and 19.32, respectively: both insignificant at the 5 per cent level, indicating that the model appears to have captured the time-dependent aspects of the excess returns process. The coefficients of skewness and kurtosis of the residuals of -0.27 and 1.88 are both significant at the 95 per cent level, suggesting that the variance equation exhibits some misspecification. The estimated conditional variance series, presented in Figure 2, illustrates the pattern in market volatility, with sizable increases in the mid-70s and at the time of the crash of 1987. With these two exceptions, the volatility of excess returns appears to have been relatively constant throughout the sample period.

Examination of a number of alternative specifications for the bond portfolio's conditional mean and variance equations led to a model of bond excess returns 
which included the contemporaneous excess returns of the stockmarket index. With a single lag in the variance equation, this $\mathrm{GARCH}(1,1)$ model yielded:

$$
\begin{aligned}
& R_{\text {Bond }_{t}}=\underset{(0.047)}{-0.037}+\underset{(0.013)}{0.064} R_{\text {Stock }_{t}}+u_{\text {Bond }_{t}} \\
& \theta_{\text {Bond }_{t}}=\underset{(0.012)}{0.034}+\underset{(0.100)}{0.332} u_{\text {Bond }_{t-1}}^{-0} \underset{(0.073)}{0.169} D_{B^{2} 40_{t-1}} \bullet u_{\text {Bond }_{t-1}}^{2}+\underset{(0.053)}{0.787} \theta_{\text {Bond }_{t-1}}
\end{aligned}
$$

The conditional variance equation for the bond portfolio's excess returns also exhibited asymmetry, but the form of the asymmetry links the magnitude of the $\mathrm{ARCH}$ effect to the sign of lagged excess returns on the bond portfolio. The dummy $D_{B 240}$ is set to unity when lagged excess returns are positive; given the dummy variable's interaction with the lagged squared error, this suggests that a negative error (excess returns falling short of that predicted by the mean equation) will have a far larger effect on the conditional variance than a positive error (excess returns larger than that predicted by the mean equation). In short, bad news matters, and the arrival of bad news will drive up the conditional variance by almost twice as much as will an element of "good news" of the same algebraic magnitude.

The estimated excess returns of the bond portfolio exhibit a strong interaction effect with contemporaneous stockmarket returns, with sizable persistence in the conditional variance series. The conditional variance of the bond portfolio exhibits a highly asymmetric response to the sign of lagged bond returns. Q statistics for the residuals and for their squares of 32.36 and 144.69 are both highly significant, indicating that unexplained time dependence remains in the series. The residual series exhibits significant skewness (coefficient of 0.36) and kurtosis (coefficient of 4.12). Figure 3 presents the estimated conditional variance series for excess returns of the bond portfolio. In contrast to the stockmarket portfolio, this series reflects a marked shift following the Federal Reserve's move to monetary targeting in October 1979. The conditional variance remains several times higher than its pre-1979 value throughout the 1979-1982 period, and only in recent years does it drift downward to levels previously experienced.

In this model, the interactions between returns in the bond markets and the stockmarket capture investors' portfolio substitution behavior between these sectors of the capital markets. While ENR essentially modelled shifts between stocks and "cash," or very short-term Treasuries, we are able to consider longer-term fixedincome investments which possess substantial market risk. 


\subsection{A dynamic model of Treasury term structure volatility}

Estimation of the models presented above for excess returns of the equallyweighted Treasury portfolio and the stockmarket portfolio has yielded two series for each of those portfolios: the predicted excess returns and the estimated conditional variances. Following Engle et al.'s methodology, these estimated series are used as predetermined variables in our models for individual maturities of Treasury securities' excess returns and conditional variances. The structure of the model implies that the individual securities' excess returns should be driven by the returns and conditional variances of a small number of common factors. In a first application, we model the security excess returns as a linear function of the two portfolios' excess return series, with a conditional variance given by a linear function of the two portfolios' conditional variances. This model (analogous to Engle et al., (1990, p.226)) may be written as:

$$
\begin{aligned}
& R_{i, t}=\lambda_{i}+\beta_{\text {Bond }_{i}} \hat{R}_{\text {Bond }_{t}}+\beta_{\text {Stock }_{i}} \hat{R}_{\text {Stock }_{t}}+\boldsymbol{V}_{i, t} \\
& v_{i, t} \mathfrak{I}_{t-1} \sim N\left(0, h_{i, t}\right) \\
& h_{i, t}=\sigma_{i}+\beta_{\text {Bond }_{i}}^{2} \hat{\theta}_{\text {Bond }_{t}}+\beta_{\text {Stock }}^{2} \hat{\theta}_{\text {Stock }}
\end{aligned}
$$

This model incorporates a constant, $\lambda$, in the mean equation, to capture nontime-varying components of the individual security's risk premium (for instance, the "on-the-run" effect that newly auctioned Treasury securities exhibit, in which their yield is driven down by demand for that specific issue). The model is estimated for each of the thirteen maturities. While the $\beta$ coefficients are highly significant for all tenors, examination of their asymptotic standard errors indicate that the direct effect of the stockmarket portfolio's excess returns and conditional variance is of considerable importance for short-term Treasury returns, but never meaningful for tenors greater than two years. In contrast to Engle et al. (1990, p.226), we found that the stockmarket index has significant direct effects on the 3-12 month segment of the yield curve.

Since the stockmarket factor does not appear to play a direct role in the individual securities' excess returns and conditional variances for medium or long tenors, we respecify the model as a single-factor model in which the estimated excess return and conditional variance of the equally-weighted bond portfolio is the only 
direct effect. This still incorporates the movements of the stockmarketportfolio, since the recursive structure of the portfolio models imply that the bond portfolio's excess returns and conditional variance estimates are derived from both the bond and stockmarket portfolios. The single-factor model estimated for individual Treasury maturities is thus:

$$
\begin{aligned}
& R_{i, t}=\lambda_{i}+\beta_{\text {Bond }_{i}} \hat{R}_{\text {Bond }_{t}}+\boldsymbol{V}_{i, t} \\
& \boldsymbol{V}_{i, t}, \mathfrak{I}_{t-1} \sim N\left(0, h_{i, t}\right) \\
& h_{i, t}=\sigma_{i}+\beta_{\text {Bond }^{2}}^{2} \hat{\theta}_{\text {Bond }_{t}}
\end{aligned}
$$

Results for this single-factor model, estimated for each of the thirteen maturities via the $\mathrm{BHHH}$ algorithm, are presented in Table $2 .{ }^{4}$ The constant term, $\lambda$, is insignificant for most tenors longer than one year. The constant in the conditional variance equation is insignificant, although a negative point estimate appears for longer tenors. The $\beta$ coefficient is highly significant for all tenors, which given the structure of the model is an indication that the restrictions embodied in a static model of excess returns as a random series with homoskedastic errors may be rejected by the GARCH alternative. For the 3-, 6-, and 9-month tenors, the restriction that the square of the $\beta$ coefficient in the mean equation appears in the conditional variance equation can be rejected. For all longer tenors, the specification of the model for individual maturities' excess returns and their conditional variance appears appropriate. It is quite apparent from the magnitude of these coefficient estimates that Treasury securities of longer maturities possess greater sensitivity to the conditional variance of the Treasury portfolio, and, given the recursive nature of the model, to the conditional variance of the stockmarket portfolio.

Simple correlations of the predicted values of our one-factor model are quite high for adjacent tenors, and decline notably as the difference in tenors increases-a quite realistic outcome, given the workings of the Treasury market. For instance, one-year and three-year securities' returns are correlated at 0.91 , but the correlation declines to 0.74 for ten year securities and 0.60 for twenty years' tenor. This feature of

\footnotetext{
4 Conceptually, the estimation of this model should take into account the "generated regressor" nature of the predicted expected return series to deal with Pagan's (1984) critique. In our context, this would necessitate maximum likelihood estimation of the full system of 13 single-factor models joint with models (2) and (3). We are investigating the feasibility of this approach, which is computationally burdensome.
} 
the model should be contrasted with the general equilibrium models of the term structure, such as that of Cox, Ingersoll and Ross (1985), which generate perfect correlations among rates at all tenors. The correlations of these predicted values are quite consistent with the original excess return series to which they are fit; for instance, the correlations between 3-month securities' excess returns and those of 1-, 2-, 5- and 20-year securities are 0.768, 0.685, 0.550, and 0.381 over the full sample, whereas the correlations among the respective predicted values are $0.76,0.67,0.54$ and 0.36. Thus, this aspect of the data-the observed comovements of the excess returns series across tenors-seems to be faithfully reflected in the one-factor model's estimates.

\section{Summary remarks}

We have implemented a variation on Engle et al.'s (1990) Factor-ARCH model for the full term structure of Treasury securities over the postwar era. The implications of their model-that a small number of common factors can successfully capture the joint behavior of securities prices over a wide range of tenors-are clearly supported by the data for short-, medium-, and long-term Treasuries. Individual tenors' returns are well explained by the innovations in a bond portfolio's returns, and their conditional variance is successfully represented as a transformation of the portfolio's conditional variance series. The interactions between volatility in the bond markets and the stockmarket are more precisely modelled in our analysis, in which we use an asymmetric GARCH approach to allow "good news" and "bad news" to have differing effects on the evolution of volatility. Despite the massive increase in Treasury rates' variance engendered by the Federal Reserve's 1979 shift in operating policy, the Factor-ARCH model captures the dynamics of the process, and is capable of dealing with the very sizable changes in bond returns generated at the long end of the term structure in response to parallel shifts in the yield curve. 


\section{References}

Canova, Fabio and Jane Marrinan, 1995. Predicting excess returns in financial markets. European Economic Review 39:1, 35-70.

Coleman, T.S., Fisher, L. Ibbotson, 1993. Historical U.S. Treasury yield curves, 19261992. New York:Moody's Investors Services.

Cox, J., J. Ingersoll and S. Ross, 1985, A theory of the term structure of interest rates, Econometrica 53, 385-467.

Engle, R.F., 1987. Multivariate ARCH with factor structures: cointegration in variance. Unpublished working paper, University of California at San Diego.

Engle, R.F., Ng, V. and M. Rothschild, 1990. Asset pricing with a Factor-ARCH covariance structure: Empirical estimates for Treasury bills. Journal of Econometrics $45,213-237$.

Engsted, T. and C. Tangaard, 1994. Cointegration and the U.S. term structure, Journal of Banking and Finance 18, 167-181.

Froot, K.A., 1989. New hope for the expectations hypothesis, Journal of Finance 44, 283-305.

Gourieroux, C. and A. Monfort, 1992. Qualitative threshold ARCH models. Journal of Econometrics 52, 159-199.

Hall, A.D., H.M. Anderson, and C.W.J. Granger, 1992. A cointegration analysis of Treasury bill yields, Review of Economics and Statistics 74, 116-126.

McCulloch, J. H. and H. Kwon, 1993. U.S. term structure data, 1947-1991.

Unpublished working paper \#93-6, Ohio State University.

Pagan, A., 1984. Econometric issues in the analysis of regressions with generated regressors. International Economic Review 25:1, 221-247.

Ross, S., 1976. Arbitrage theory of capital asset pricing. Journal of Economic Theory 13:341-360. 
Table 1: Descriptive Statistics of Excess Returns Series

\begin{tabular}{|c|c|c|c|c|c|c|}
\hline Series & Mean & Variance & Skewness & Kurtosis & $\mathrm{Q}(12)$ & QSQR(12) \\
\hline 3 Month & 0.081 & 0.016 & 2.165 & 11.159 & 95.512 & 103.433 \\
\hline 6 Month & 0.091 & 0.078 & 1.590 & 10.733 & 51.387 & 144.593 \\
\hline 9 Month & 0.101 & 0.188 & 1.188 & 9.558 & 45.415 & 145.273 \\
\hline 12 Month & 0.097 & 0.350 & 0.956 & 9.373 & 43.431 & 146.538 \\
\hline 18 Month & 0.119 & 0.737 & 0.668 & 8.954 & 45.069 & 132.509 \\
\hline 2 Year & 0.112 & 1.258 & 0.589 & 9.161 & 40.898 & 123.192 \\
\hline 3 Year & 0.130 & 2.296 & 0.128 & 7.527 & 32.807 & 115.507 \\
\hline 4 Year & 0.115 & 3.746 & 0.012 & 6.741 & 24.013 & 110.691 \\
\hline 5 Year & 0.124 & 4.823 & 0.010 & 5.272 & 22.547 & 123.290 \\
\hline 7 Year & 0.107 & 7.720 & 0.054 & 3.379 & 18.064 & 151.013 \\
\hline 10 Year & 0.073 & 12.633 & 0.274 & 2.954 & 24.061 & 174.612 \\
\hline 15 Year & 0.007 & 26.737 & 0.476 & 3.161 & 28.163 & 147.441 \\
\hline 20 Year & -0.103 & 41.007 & 0.041 & 2.969 & 23.879 & 107.062 \\
\hline VW Stock & 0.453 & 19.793 & -0.330 & 2.269 & 12.304 & 14.069 \\
\hline EW Bond & 0.081 & 3.697 & 0.343 & 3.817 & 32.316 & 150.908 \\
\hline
\end{tabular}

Notes: $\mathrm{Q}(12)$ is the Ljung-Box statistic for 12th order serial correlation in the excess returns series. QSQR(12) is the equivalent statistic for the squares of the excess return series. VW Stock is the valueweighted index of NYSE / AMEX equities. EWB is an equal-weighted portfolio of the Treasury tenors. Statistics are calculated over the monthly observations 1962:7-1992:12.

Table 2: One-Factor Model for Individual Treasury Maturities

$$
R_{i, t}=\lambda_{i}+\beta_{\text {Bond }_{i}} \hat{R}_{\text {Bond }_{t}}+\boldsymbol{V}_{i, t}, \quad \boldsymbol{V}_{i, t}\left(\mathfrak{I}_{t-1} \sim N\left(0, h_{i, t}\right), \quad h_{i, t}=\sigma_{i}+\beta_{\text {Bond }_{i}}^{2} \hat{\theta}_{\text {Bond }_{t}}\right.
$$

\begin{tabular}{|c|c|c|c|c|c|c|c|c|c|c|c|c|c|}
\hline & mo & $6 \mathrm{mo}$ & $\mathrm{mo}$ & $12 \mathrm{mo}$ & $18 \mathrm{mo}$ & $2 \mathrm{yr}$ & $3 \mathrm{yr}$ & $4 \mathrm{yr}$ & $5 \mathrm{yr}$ & $7 \mathrm{yr}$ & $10 \mathrm{yr}$ & $15 \mathrm{yr}$ & $20 \mathrm{yr}$ \\
\hline$\lambda$ & 040 & 050 & 043 & 033 & 0.027 & 0.008 & 0.008 & .015 & .001 & & .061 & .122 & .309 \\
\hline $\operatorname{se}(\lambda)$ & 004 & .009 & 013 & 0.018 & 0.026 & 0.035 & 0.043 & 0.054 & 0.063 & 0.084 & 0.104 & 0.150 & 0.242 \\
\hline & 067 & 0.139 & 0.214 & 0.292 & & 0.571 & 0.808 & .044 & 183 & 1.505 & 1.884 & 2.691 & 3.692 \\
\hline & 002 & 0.005 & & & & 0.022 & 0.028 & & & & & & 0.141 \\
\hline$\sigma$ & & 001 & & & & & & & & & & & .063 \\
\hline & 0.00 & & & & & & & & & & & & 0.721 \\
\hline & 0.016 & 0.076 & 0.180 & 0.334 & 0.699 & 1.194 & 2.174 & 3.556 & 4.565 & 7.303 & 11.825 & 24.818 & 37.948 \\
\hline Ske & 171 & 1.661 & 1.239 & 0.986 & 0.679 & 0.592 & 0.119 & 003 & & 0.070 & 0.279 & 0.487 & 0.080 \\
\hline & 11.079 & 10.931 & 9.860 & 9.704 & 9.363 & 9.591 & 7.917 & 7.061 & 5.508 & 3.443 & 3.026 & 3.355 & 3.396 \\
\hline & 0.11 & 2.57 & 47.07 & 44. & & 41 & 32.45 & 3.30 & & & & 70 & 22.51 \\
\hline & & & & & & & & & & 33 & 21 & 06 & 0.032 \\
\hline QS(12 & 93.41 & 149.22 & 150.32 & 149.22 & 134.13 & 122.97 & 113.84 & 107.39 & 120.15 & 148.55 & 165.88 & 125.69 & 93.45 \\
\hline signif & 0.000 & .000 & 0.000 & 0000 & 0.000 & 0.000 & 0.000 & 0.000 & 0.000 & 0.000 & 0.000 & 0.000 & 0.000 \\
\hline
\end{tabular}

Notes: asymptotic standard errors are given below the coefficient estimates. Var, Skew and Kurt are the variance, skewness, and kurtosis of the residuals. Q(12) is the Ljung-Box statistic for 12th order serial correlation in the residuals. QS(12) is the equivalent statistic for the squares of the residual series. 
Figure 1: Moving Correlations among Excess Returns on Treasury Securities

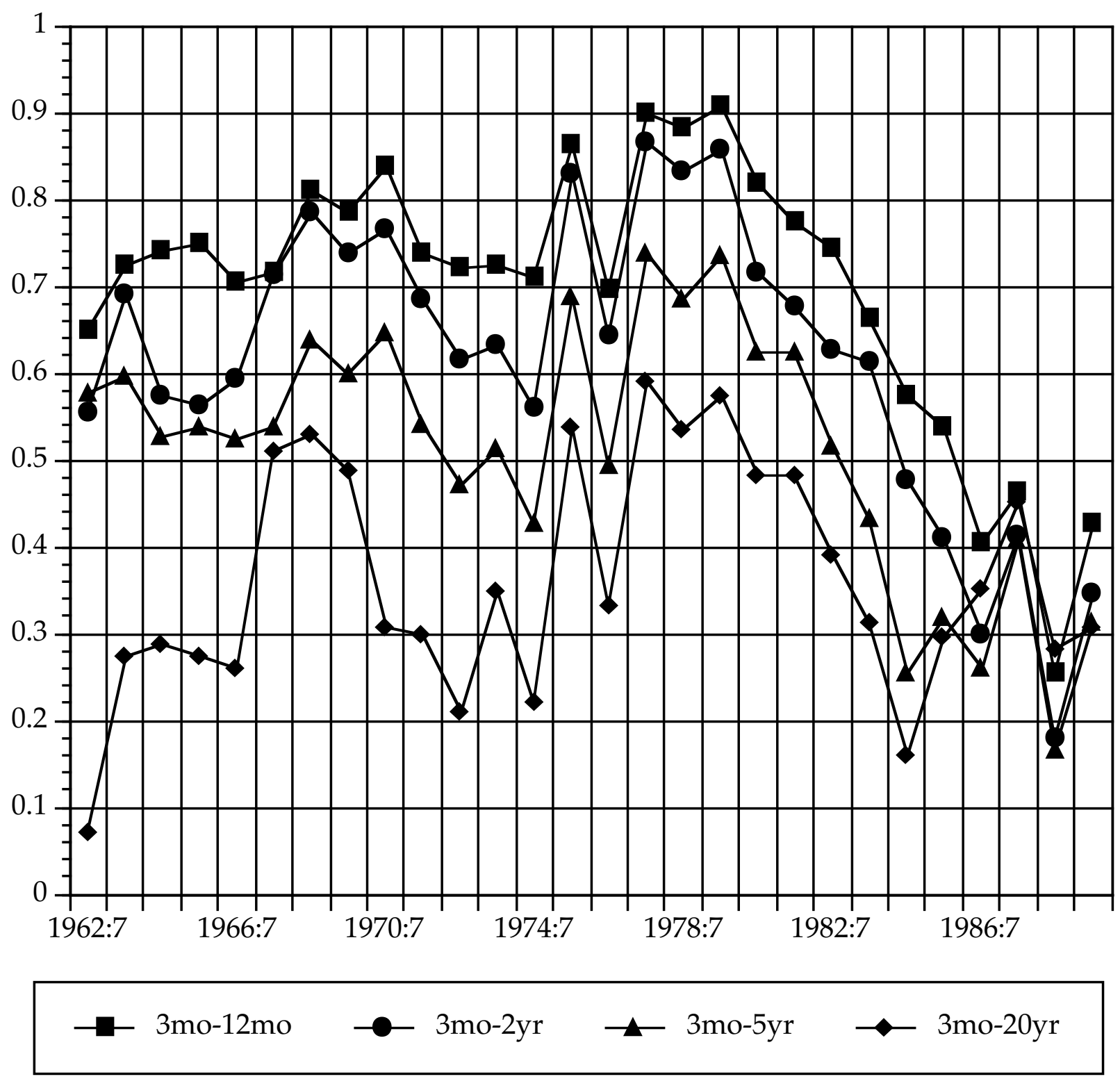

Note: simple correlations computed annually from 36 observations. 
Figure 2: Estimated Conditional Variance of Stock Portfolio

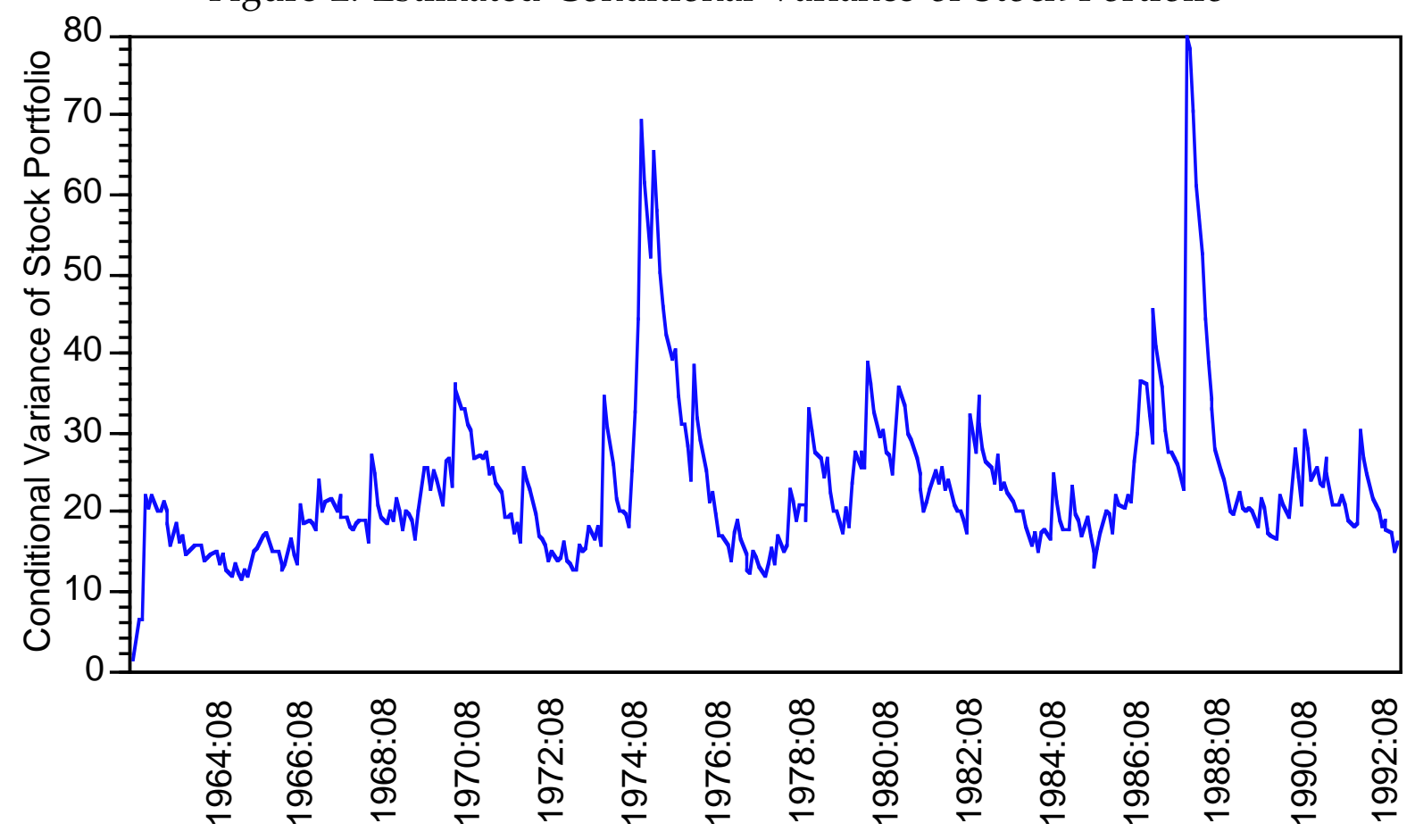

Figure 3: Estimated Conditional Variance of Equally-Weighted Treasury Portfolio

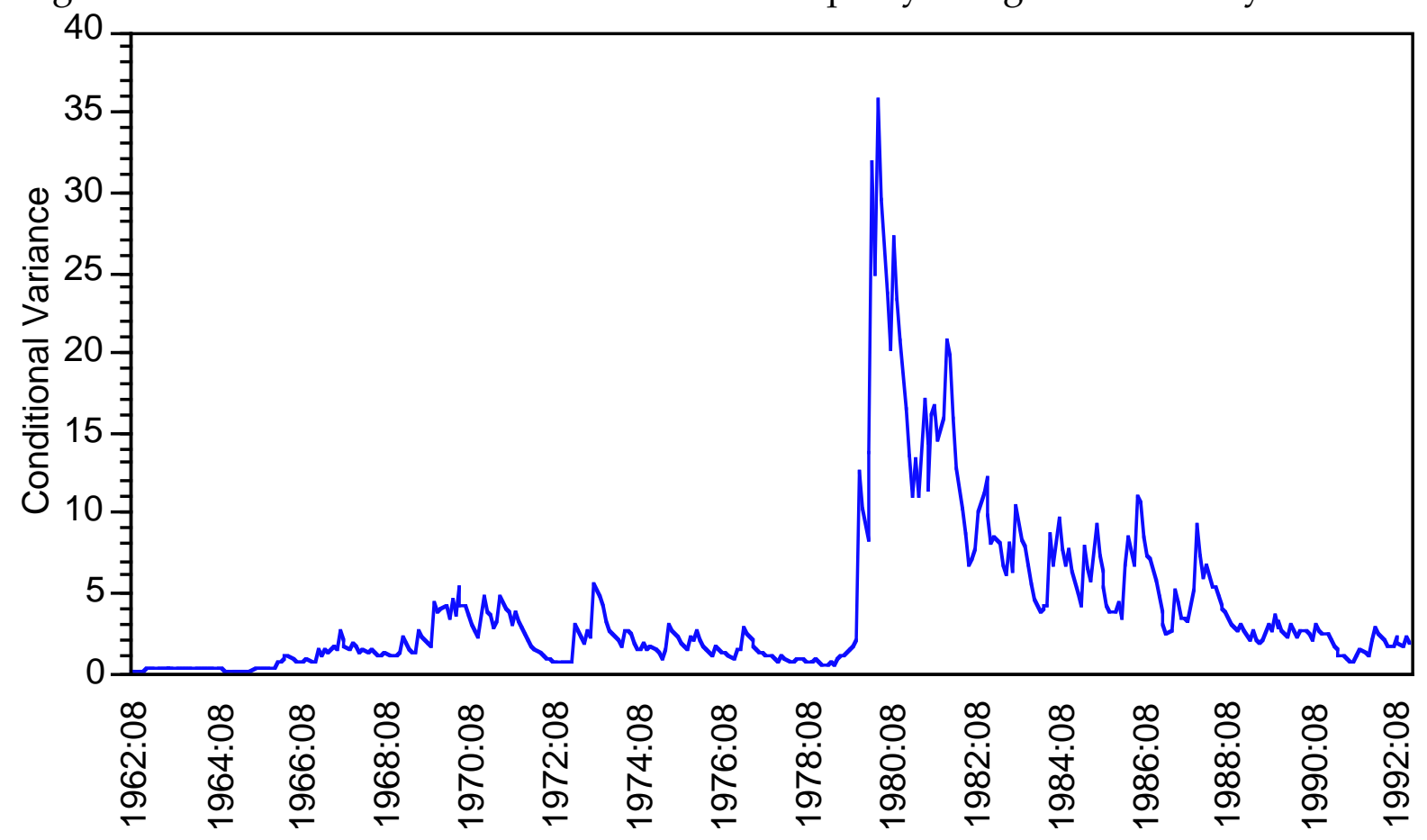

